\title{
Tradução e adaptação semântica para versão em português do Stressful Life Events Schedule (SLES)
}

\author{
Cristian Patrick Zeni - Universidade Federal do Rio Grande do Sul, Porto Alegre, Brasil \\ Roberta Paula Schell Coelho - Pontifícia Universidade Católica do Rio Grande do Sul, Porto Alegre, Brasil \\ Aline Aparecida Martins Ferreira - Pontifícia Universidade Católica do Rio Grande do Sul, Porto Alegre, Brasil \\ Priscila de Oliveira Machado - Pontificia Universidade Católica do Rio Grande do Sul, Porto Alegre, Brasil \\ Silzá Tramontina - Universidade Federal do Rio Grande do Sul, Porto Alegre, Brasil \\ Rodrigo Grassi-Oliveira - Pontifícia Universidade Católica do Rio Grande do Sul, Porto Alegre, Brasil
}

\begin{abstract}
Resumo
O Stressful Life Events Schedule (SLES) é um instrumento que visa investigar a presença e o impacto de eventos de vida estressantes (EVE) ocorridos nos últimos 12 meses. O objetivo deste trabalho é traduzir e adaptar por equivalência semântica os itens da escala para a língua portuguesa. Seis etapas foram realizadas: (1) Tradução; (2) Retrotradução; (3) Revisão técnica e adaptação semântica; (4) Avaliação e validação do conteúdo e do significado geral considerando o contexto da população; (5) Realização de uma medida de confiabilidade em relação ao construto dependência/independência dos EVE; e (6) Produção da versão final do instrumento. O tempo verbal se manteve na maioria dos itens da escala. Algumas alterações foram necessárias como um acréscimo da variação de alguns termos. A versão adaptada demonstrou ser de fácil aplicação, não tendo sido avaliada como extensa e contemplando um número considerável de EVE.

Palavras-chave: Experiências de vida, Transtorno bipolar, Tradução, Linguagem.
\end{abstract}

Translation and semantic adaptation for the version in Portuguese of the Stressful Life Events Schedule

\begin{abstract}
The Stressful Life Events Schedule (SLES) is an instrument to investigate the presence and impact of stressful life events (SLE) occurred during the latest 12 months. The aim of this study is to translate and adapt, through semantic equivalence, the items from the instrument to the Portuguese language. The process had six steps: (1) Translation; (2) Backtranslation; (3) Technical review and semantic adaptation; (4) Assessment and content validation and overall meaning, considering the context of the population; (5) A measure of reliability was conducted in relation to the dependence/ independence construct; and (6) Production of the final version of the instrument. The verbal conjugation was maintained in most of the scale's items. Some changes were required, such as an addition of the variation of some terms. The adapted version proved to be of easy application, not too extensive and contemplating a considerable number of SLE.

Keywords: Life events, Bipolar disorder, Translation, Language.
\end{abstract}

Traducción y adaptación semántica de la versión en portugués del Stressful Life Events Schedule (SLES).

\section{Resumen}

El Stressful Life Events Schedule (SLES) es una escala que objetiva investigar la presencia y el impacto de los acontecimientos vitales estresantes (EVE) en los últimos 12 meses. El objetivo del presente estudio es traducir y adaptar por equivalencia semántica los ítems de la escala para la lengua portuguesa. Las seis etapas realizadas fueron: (1) traducción y (2) retraducción, (3) revisión técnica y adaptación semántica, (4) evaluación y validación del contenido y del significado general teniendo en cuenta el contexto de la población, (5) realización de una medida de fiabilidad para el constructo dependencia / independencia de los EVE, y (6) producción de la versión final del instrumento. El tiempo verbal se mantuvo en la mayoría de los ítems de la escala. Algunas modificaciones fueran necesarias, tales como el aumento de la variación de algunos términos. La versión adaptada se mostró de fácil aplicación, no evaluada como extensa y contemplando un número considerable de EVE.

Palabras clave: Experiencias de vida, Trastorno bipolar, Traducción, Lenguaje.

As etapas iniciais do desenvolvimento são um período crucial no qual ocorrem o máximo crescimento cerebral e a formação dos padrões de regulação emocional que influenciam nos desfechos de saúde mental (Shonkoff, Boyce \& McEwen, 2009). Pouco se sabe ainda sobre a etiologia dos transtornos mentais ao longo da vida, e o cenário atual da saúde mental na infância e adolescência é alarmante. Em torno de $10-20 \%$ de crianças e adolescentes ao redor do mundo apresentam um ou mais quadros psicopatológicos (Kieling e cols., 2011). Os estudos científicos têm abordado fatores ambientais e biológicos (Arseneault e cols.,2011; Danese e cols., 2009; Polanczyk e cols., 2009), porém sem conclusões definitivas.

Estudos em pesquisa básica e avançada revelam que as experiências precoces adversas podem ter efeitos duradouros sobre o organismo, no sistema de resposta ao estresse, em estruturas cerebrais e no processamento emocional (Crowe \& Blair, 2008; De Bellis \& Van Dillen, 2005; Pine, 2007). Abre-se uma gama de questionamentos acerca do impacto do 
ambiente na formação do indivíduo, como e o quanto as experiências precoces podem interferir no seu desenvolvimento.

Os primeiros estudos com o uso de instrumentos padronizados na associação entre eventos de vida e psicopatologia em crianças e adolescentes datam do início da década de 70 (Brown, Sklair, Harris \& Birley, 1973; Coddington, 1972a; Coddington, 1972b). Desde então, vem se buscando a superação das limitações encontradas em torno deste tipo de instrumento de avaliação, tais como: o número finito de eventos de vida, os níveis de subjetividade, tempo de exposição, grau de gravidade. Inicialmente, os instrumentos eram predominantemente baseados em métodos de listagem de itens (checklists) autopreenchidos.

$\mathrm{O}$ primeiro instrumento desenvolvido sobre eventos de vida para aplicação em crianças e adolescentes foi denominado Life Event Record (LER) (Coddington, 1972a). A partir da LER, foi gerada a Life Events Checklist (LEC), a qual continha 46 eventos e acrescentaram-se espaços para que o respondente pudesse acrescentar demais eventos ocorridos que não constassem na lista (Johnson \& McCutcheon,1980). Além disso, o sujeito deveria indicar se o evento era positivo ou negativo, o grau de incômodo e o estresse provocado. Esses instrumentos foram amplamente publicados em estudos associando eventos adversos e diversas psicopatologias (Banez \& Compas, 1990; Kashani, Vaidya, Soltys, Dandoy \& Reid, 1990; Williamson e cols. 1995; Williamson, Birmaher e cols. 1998).

Em 1973, Brown e colaboradores refinaram seus estudos e desenvolveram uma entrevista acompanhada de escala para adultos denominada Bedford College Life Event and Dificulty Schedule (LEDS), que contemplava a ocorrência da data do evento, cálculo do tempo de exposição, o grau de ameaça percebido, escores para dimensão e tipo do evento (perda, risco), incluindo a atribuição do sujeito, se o evento dependia ou não do seu comportamento. A maior limitação encontrada na aplicação deste instrumento foi o tempo de aplicação, de aproximadamente 30 horas de entrevista.

Dentre os vieses avaliados, pesquisadores engajam-se para um maior entendimento sobre $\mathrm{o}$ impacto dos eventos negativos ao longo da vida (Danese e cols., 2009; Kieling, e cols., 2001; Salum, e cols., 2010). Os eventos de vida estressantes (EVE) ocorridos durante a infância e adolescência podem ser classificados como uma exposição a uma única ou a diferentes situações que causam estresse no organismo e que excedem a capacidade da criança ou adolescente de compreender e lidar com esse evento. Entre os eventos estão situações como: abuso físico, emocional, verbal ou sexual, negligência, testemunho de violência, privação social, perda ou separação parental, pobreza, uso de drogas e até mesmo catástrofes ambientais (Brown e cols., 2009; Pechtel \& Pizzagalli 2011).

Pesquisas também têm mostrado que o acúmulo de EVE no início da vida podem contribuir para o aparecimento de psicopatologia ao longo do desenvolvimento (Angst, Gamma, Rössler, Ajdacic \& Klein, e cols., 2011; Horesh \& Iancu 2010; Sar e cols., 2010). Os eventos estressantes ocorridos no início do período desenvolvimental estão relacionados a danos neurodesenvolvimentais, podendo causar alterações neuropsicológicas, como déficits cognitivos na memória e aprendizagem (Carrion, Weems \& Reiss, 2007; De Bellis, Hooper, Woolley \& Shenk, 2010).

Estudos referem que crianças com história de trauma e provenientes de um ambiente familiar adverso apresentam maior vulnerabilidade ao desenvolvimento de transtorno de estresse pós-traumático (Bendall, e cols. 2012; Carter, e cols., 2006). Além disso, crianças que vivenciam EVE apresentam mais dificuldades com os pares e problemas emocionais, além de um maior acometimento de determinadas psicopatologias, como ansiedade e depressão (Copeland, Keeler, Angold \& Costello, 2007; Flouri \& Panourgia, 2011). Também há evidências de que a presença de sintomas, mesmo sem o preenchimento de critérios para o transtorno de estresse pós-traumático (TEPT), pode indicar efeitos e prejuízos do trauma em crianças (Copeland e cols., 2007). A investigação de EVE em crianças e em adolescentes abarcando um delineamento longitudinal é fundamental para responder a algumas questões sobre fatores relacionados ao surgimento e ao curso de diversas psicopatologias (Dohrenwend, 2006).

Williamson e colaboradores compararam 45 crianças com depressão, 20 com transtorno de ansiedade e 11 crianças controles sem patologia psiquiátrica, usando o LER, e encontraram que o grupo com depressão, particularmente as meninas, estava mais exposto a eventos traumáticos em relação ao grupo controle (Williamson, Birmaher, Dahl \& Ryan, 2005). Estudos em populações clínicas têm mostrado que os adolescentes com depressão unipolar apresentam um número significativamente maior de eventos de vida estressantes do que adolescentes sem psicopatologia e, até mesmo, apresentam mais eventos do que adolescentes com um transtorno de ansiedade (Mayer e cols., 2009; Williamson e cols., 2005).

Numa perspectiva epidemiológica prospectiva, Pine e colegas avaliaram 776 jovens americanos em 1986 acerca dos seus eventos de vida, através da Life Event Record (Coddington, 1972b), e utilizaram a Diagnostic Interview Schedule for Children (Costello, Edelbrock \&Costello, 1985) para uma avaliação psicopatológica. Em 1992, os sujeitos refizeram a 
avaliação psiquiátrica. Mediante uma análise de regressão linear, na qual os eventos de vida foram tratados como uma variável independente, os dados revelaram que o número total de eventos de vida durante a adolescência prevê um aumento no risco de desencadeamento de episódios de depressão e ansiedade na adultez (Pine, Cohen, Johnson \& Brook, 2002).

Romero e cols. (2009) realizaram um estudo para verificar as taxas de ocorrência de EVE entre os seguintes grupos: 446 jovens entre 7 e 17 anos com transtorno do humor bipolar tipos I $(\mathrm{n}=260)$, II $(\mathrm{n}=32)$ e sem outra especificação (SOE) $(n=154) ; 65$ jovens com transtorno depressivo e/ou ansioso, conforme os critérios da DSM-IV; e 65 jovens controles sem psicopatologia. Todos os sujeitos preencheram o Life Events Checklist (Johnson \& McCutcheon,1980) e foram entrevistados por meio da Schedule for Affective Disorders and Schizophrenia for School-Age Children-Present and Lifetime version (K-SADS-PL) (Kaufman e cols., 1997). Os achados revelaram que tanto os jovens com depressão e/ou ansiedade quanto os jovens bipolares estão expostos a um número maior de EVE, sendo eles independentes e dependentes, o que pode levar a implicações em longo prazo e consequências negativas num desfecho associadas com esses transtornos.

De acordo com a relevância dos eventos de vida no desenvolvimento e a repercussão que esses podem acarretar para o indivíduo, percebeu-se a necessidade de um instrumento que servisse como auxilio na coleta de dados sobre as experiências vitais, tanto para fins de pesquisa, como para aplicação clínica no nosso idioma. A literatura no tema é ampla, porém observa-se ainda a escassez de estudos na nossa população que utilizem um instrumento que abarque o impacto dos EVE (Williamson e cols., 2003, 2005).

Diante essa limitação e baseados na literatura sobre avaliação de EVE na infância e adolescência, Williamson e colaboradores (2003) utilizaram-se dos pontos fortes do instrumento denomidado Life Events and Difficulties Schedule (LEDS) e de listas de eventos de vida auto aplicadas, como a Life Events Checklist (LEC) e desenvolveram um instrumento denominado Stressful Life Events Schedule (SLES).

A SLES (Williamson et al, 2005) é uma entrevista face a face que investiga EVE ocorridos nos últimos 12 meses. Essa entrevista é acompanhada por uma escala contendo determinados eventos estressores e uma escala likert de quatro pontos (nem um pouco, um pouco, bastante e muito) para verificar a intensidade do impacto desses eventos para o sujeito. Essa entrevista comporta três cadernos: um para ser utilizado em crianças até 12 anos, contendo 61 eventos; outro em adolescentes a partir de 12 anos, contendo 79 eventos; e um para ser utilizado com os pais e/ou responsáveis, com 79 questões. O preenchimento dessa escala pelos pais objetiva uma coleta de dados mais completa e precisa. Os eventos são classificados dentre aqueles que têm maior probabilidade de ocorrer de forma independente (por exemplo, morte de um familiar) ou dependente (por exemplo, desempenho escolar) sobre o comportamento da criança ou adolescente (Williamson e cols., 2005).

No estudo de desenvolvimento do instrumento, todas as crianças e adolescentes foram inicialmente entrevistados presencialmente com o SLES a respeito da ocorrência de eventos estressantes durante o ano anterior à entrevista. $\mathrm{O}$ formato atual de entrevista do SLES envolve perguntar sistematicamente ao informante se cada um dos eventos ocorreu e, caso a resposta seja positiva, algumas perguntas posteriores são realizadas a fim de investigar mais profundamente informações relevantes para acessar dados do evento. Por exemplo, na sessão de moradia, perguntava-se aos informantes se eles mudaram de residência durante $\mathrm{O}$ ano anterior. Caso tivessem mudado, as seguintes questões seriam realizadas: Por que você se mudou? Você queria se mudar? Você teve que mudar de escola também? Você gosta de sua nova casa? Você se sente muito longe de seus amigos, nesta nova casa? Você prefere morar em outro lugar?

Estressores contínuos que estavam presentes durante o ano anterior, mas tinham começado antes mesmo do tempo de início da entrevista foram datados a partir de quando começaram (p. ex.: uma criança que afirmasse ter presenciado brigas dos pais no ano anterior, conforme solicita a entrevista, também era questionada sobre quando estas brigas haviam iniciado para datar o tempo de exposição aos eventos estressantes). Para controlar o viés de memória, metade da amostra foi escolhida aleatoriamente para completar a LEC antes de serem entrevistados com o SLES e a outra metade da amostra completou a LEC depois de ser entrevistado com o SLES (Hulley e cols., 2001).

Depois dos filhos serem entrevistados, todos os pais foram entrevistados presencialmente com o SLES sobre os eventos de vida estressores que ocorreram na vida de seus filhos durante o último ano pelo mesmo entrevistador. A fim de examinar a validade convergente entre o SLES e o Life Events and Difficulties Schedule (LEDS), 30 jovens (15 com psicopatologia e 15 controles normais) foram selecionados aleatoriamente dentro de cada um dos grupos e entrevistados com os LEDS, e seu responsável entrevistado com o SLES. A LEDS foi sistematicamente administrada por último, a fim de que qualquer potencial viés de memória permanecesse a favor do mesmo, aumentando assim as chances de que ele obtivesse um melhor desempenho 
em comparação com o SLES. Estava previsto que a exposição inicial ao LEC e ao SLES pudesse auxiliar os entrevistados a lembrar mais claramente dos eventos que ocorreram durante o ano anterior, bem como aumentar a precisão com que eles datavam os eventos. A etapa final do estudo envolveu uma segunda entrevista com todas as crianças e adolescentes utilizando o SLES. Em média, a segunda entrevista (reteste) foi realizada com a variação de 5 a 15 dias após a entrevista inicial do SLES (Williamson e cols., 2003).

O presente trabalho tem como objetivo traduzir para a língua portuguesa o SLES desenvolvido por Williamson e cols. (2003) e explicitar os procedimentos efetuados na adaptação transcultural por meio de equivalência semântica dos itens tanto para $\mathrm{O}$ significado geral, como para o referencial.

\section{Método}

O processo de tradução e adaptação foi realizado por meio da metodologia proposta por Pasquali (2000), em seis etapas consecutivas.

$\mathrm{Na}$ etapa 1 fez-se a tradução da versão original inglês (VO) do instrumento para o português, gerando a versão 1 (V1). Esta foi realizada por um Psiquiatra da Infância e Adolescência brasileiro, proficiente e fluente em língua inglesa (CPZ). $\mathrm{Na}$ etapa 2, foi realizada a retrotradução (R1) da V1 ao inglês por um profissional bilíngüe (AMF), cuja língua nativa é o português. $\mathrm{Na}$ etapa 3, um psiquiatra (RGO) e uma psicóloga (RPSC), de forma independente, fizeram uma revisão técnica e julgamento de equivalência semântica do significado geral e referencial dos termos e das expressões de todos os itens que compõem a escala. Nesta etapa, manteve-se a equivalência semântica entre a primeira e a segunda tradução, sob a perspectiva do significado referencial dos termos/palavras originais.

Os significados referenciais mencionam a equivalência literal entre as palavras e expressões. Acredita-se que, se o significado referencial é o mesmo no original e na tradução, existindo correspondência literal entre eles (Pasquali, 2000). Priorizou-se também o significado geral de cada item do instrumento referido na tradução em relação ao original. $O$ significado geral é muito relevante, na medida em que a tradução literal de uma palavra não implica, necessariamente, uma mesma evocação subjetiva vivenciada em diferentes culturas. É fundamental considerar a implicação e significado que as palavras e expressões têm nas diversas culturas, assim como os diferentes níveis educacionais e socioeconômicos. Dessa forma, o significado geral refere-se à adequação e aceitabilidade do estilo empregado ou o uso específico de um termo nos itens do instrumento (Jorge, 2000).

$\mathrm{Na}$ etapa 4, para se avaliar a validação do conteúdo e o significado geral dentro do contexto da população, a versão corrigida foi apresentada para cinco juízes, que analisaram as versões original, a traduzida e retrotraduzida. Os processos de tradução e retrotradução foram encaminhados para esse comitê de juízes, composto por uma equipe multidisciplinar especializada (dois psiquiatras, duas psicólogas e uma assistente de pesquisa em psicologia), conhecedores da finalidade do instrumento e do tema a ser analisado. Foi solicitado que lessem o instrumento, verificassem item por item, e sugerissem modificações. Caso a linguagem não parecesse adequada, pedia-se que se anotasse também a razão dessa observação para a detecção de possíveis divergências nas traduções. Além disso, pediu-se que fosse verificado se os itens da escala eram pertinentes ao tema que mensuravam. A validade de conteúdo refere-se à extensão em que o conteúdo de um teste está realmente abrangendo as suposições teóricas sobre o seu objeto de interesse e a não contenção de aspectos que possam ser atribuídos a outros objetos, verificando assim se as dimensões medem e abarcam aquilo que o instrumento tem a intenção de avaliar.

A validade de conteúdo considera essencialmente a precisão do conteúdo avaliado, geralmente mediante um consenso entre especialistas e da adequação dos itens propostos na escala. $\mathrm{Na}$ etapa 5 , realizou-se uma medida de confiabilidade com o intuito de analisar o nível de concordância entre três avaliadores em relação ao construto dependência/independência dos EVE. E, na etapa 6, baseados então na opinião dos juízes, produziu-se a versão final do instrumento (VF). Em razão do grande número de itens contemplados no instrumento, não foi possível explicitá-lo qualitativamente neste artigo. Portanto, caso haja interesse, contatar o autor para posterior envio. As sugestões ofertadas pelos juízes contemplaram uma modificação de nove itens nesse instrumento de 79 questões. Foi observada uma necessidades de alteração de $11,39 \%$ dos itens a fim de melhor adaptá-lo para o entendimento da nossa população.

\section{Resultados}

Em geral, as sentenças traduzidas mantiveram-se no pretérito perfeito, mesmo tempo verbal das originais. $\mathrm{Na}$ tradução da escala likert, optou-se por empregar termos de quantificação frequentemente mais utilizados para um melhor entendimento. A escala original é dividida em quatro termos: Not at all, A little, Somewhat e A lot, sendo traduzida como Nem um 
pouco, Um pouco, Bastante e Muito, respectivamente. Uma das alterações também realizada foi o acréscimo da variação dos termos do instrumento, para abranger os dois gêneros, feminino e masculino, por exemplo, abusado(a).

A sentença I was sexually hurt or touched in private parts, foi traduzida como Eu fui machucado(a) ou tocado(a) sexualmente em minhas partes íntimas, pois partes privadas, que seria a terminologia traduzida, é menos utilizada do que partes íntimas. Outro item como My home was damaged of fire, flood, storm, tornado or other event, a tradução foi modificada para Minha casa foi danificada por causa de incêndio, enchente, tempestade, ventania, ou outros eventos, pois tornados não são comuns na realidade brasileira. $\mathrm{O}$ item I broke off an engagement foi adaptado para o português como Eu terminei um relacionamento, pois, dentro da faixa etária-alvo da escala, noivados são pouco frequentes no Brasil. Na sentença I was hurt or punched by someone a versão brasileira ficou como Eu fui machucado ou apanhei de alguém, para melhor compreensão da frase pelo público-alvo. A sentença I was bullied at school or in my neighborhood, foi modificada para Debocharam de mim na escola ou vizinhança, pois esta é uma palavra sem uma tradução definitiva, mas entende-se que a natureza do bullying seria de um tratamento abusivo para com aquela pessoa. A frase I started my menstrual cycle (period) foi adaptada para Eu menstruei pela primeira vez, para que houvesse maior equivalência de estilo, pois a expressão começar um ciclo menstrual é de pouco uso na população geral. Em I did badly on a big test, a tradução ficou como Tive maus resultados em um teste importante a fim de manter o significado original da frase, mesmo esta não sendo uma tradução literal da sentença original.

Para garantir, ainda, a confiabilidade do construto independência/dependência dos eventos, ou seja, aqueles eventos que eram dependentes do comportamento do adolescente, e eventos que independiam do mesmo, foi realizada uma medida de confiabilidade entre observadores, seguindo os mesmos procedimentos usados na construção do instrumento original. Três dos autores de forma independente (R. P. S. C - (A), A. A. F - (B), P. O. M (C)), classificaram cada um dos eventos como independentes do comportamento do adolescente (por ex., a morte de um familiar, separação dos pais) ou dependente do comportamento do adolescente (por ex., mau desempenho escolar).

Realizamos, então, a medida kappa, com o intuito de analisar o nível de concordância entre os avaliadores em relação à dependência/independência entre sujeito e o fator estressante de vida. Nesse método, as medidas variam entre 0 e 1 , sendo 0 um índice esperado para uma concordância observada ao acaso e 1 o encontrado em uma concordância perfeita. Esses resultados foram então comparados entre si e resultaram em três duplas de avaliadores, para cada tipo de protocolo utilizado. Os resultados de concordância obtidos, que estão na tabela a seguir, variaram entre concordância substancial e boa, o que, para a natureza da escala, é satisfatório. Esses índices de concordância se assemelharam aos apresentados no estudo original do desenvolvimento do instrumento, conferindo assim mais robustez para este estudo (Tabela 1)

Tabela 1. Medida kappa entre Avaliadores

\begin{tabular}{lccccccccc}
\hline $\begin{array}{l}\text { Protocolo SLES } \\
\text { Avaliadores }\end{array}$ & $\mathrm{AB}$ & Criança & \multicolumn{3}{c}{ Adolescente } & \multicolumn{3}{c}{ Pais } \\
\hline ED-ED & 16 & 15 & 14 & 33 & 28 & 30 & 28 & 26 & 26 \\
ED-EI & 4 & 7 & 7 & 6 & 11 & 9 & 11 & 10 & 9 \\
EI-EI & 41 & 39 & 40 & 40 & 40 & 40 & 40 & 43 & 44 \\
Kappa & 0,84 & 0,73 & 0,72 & 0,84 & 0,72 & 0,77 & 0,72 & 0,73 & 0,76 \\
Concordância & $\mathrm{B}$ & $\mathrm{S}$ & $\mathrm{S}$ & $\mathrm{B}$ & $\mathrm{B}$ & $\mathrm{S}$ & $\mathrm{S}$ & $\mathrm{S}$ & $\mathrm{S}$ \\
\hline
\end{tabular}

Nota: ED- evento dependente; EI- evento independente; S- substancial; B-boa

Discussão

A literatura tem apresentado uma grande diversidade de métodos para avaliar eventos de vida (Mayer e cols.,2009; Pine e cols., 2002; Romero e cols., 2009; Williamson e cols., 2003, 2005;). Essa heterogeneidade metodológica dificulta o seguimento de uma diretriz para abordagem do tema. Apesar de apresentarem uma significativa relevância tanto para aplicação clínica, como em pesquisa, nacionalmente ainda existem poucos instrumentos confiáveis e adequados para avaliar EVE em crianças $e$ adolescentes. $\mathrm{O}$ presente estudo apresentou a tradução e avaliação da equivalência conceitual, semântica e de itens do SLES, que se mostrou uma ferramenta útil na investigação de EVE.

A construção da versão original teve o propósito de dar conta das limitações apresentadas pelos demais 
métodos de avaliação já existentes, focando-se assim em adequar o tempo de aplicação, abranger um maior número de EVE, investigar a gravidade do evento percebida pelo respondente ter uma entrevista face a face para o melhor preenchimento do instrumento. Dessa forma, após a verificação da boa qualidade psicométrica apresentada pelo SLES, optou-se por traduzi-lo e adaptá-lo.

De modo geral, os itens obtiveram boa equivalência entre as versões. A apreciação da equivalência conceitual e de itens propiciou ajustes linguísticos e adaptações terminológicas de alguns termos. De acordo com a avaliação realizada pelos juízes, foi sugerida uma modificação de $11,39 \%$ dos itens da escala. Esse dados reiteram e apontam a importância de estudos de tradução e adaptação semântica dos instrumentos na área de avaliação.

\section{Considerações finais}

Os estudos voltados para o levantamento da ocorrência de eventos de vida em etapas precoces do desenvolvimento são fundamentais, na medida em que alertam para a repercussão que os fatores ambientais têm na saúde mental a longo prazo. É importante que os estudos se voltem para o rastreio desses ambientes estressantes comuns entre os transtornos mentais, almejando, assim, uma melhor compreensão dessa associação.

É importante que os pesquisadores estejam atentos e ambicionando substituir os gastos atuais em pesquisas na área de avaliação e intervenção para focarem seus investimentos na área preventiva. Para tanto, é necessária a realização de estudos aliando padrões de resposta ao estresse e capacidade de resiliência à exposição a eventos de vida e fatores neurobiológicos, já que a vivência de experiências precoces adversas vem sendo associada com alterações no processamento emocional e no sistema de resposta ao estresse (Crowe \& Blair, 2008; De Bellis \& Van Dillen, 2005; Pine, 2007). Dessa forma, pesquisas longitudinais de coorte são necessárias para haver maior robustez no poder preditivo da direção de causalidade entre ambiente adverso e psicopatologia.

A partir de um maior aprofundamento nos fatores ambientais intervenientes na psicobiologia, buscar-se-á um enfoque em intervenção precoce tendendo-se evitar o aumento do crescimento de indivíduos acometidos por psicopatologias. Além disso, futuros estudos são necessários para avaliar as demais propriedades psicométricas do SLES na nossa cultura.

As limitações sugeridas por este estudo referem-se novamente à falta de estudos com o mesmo instrumental para traçarmos comparações e propormos futuros refinamentos. Embora a escala abranja um número razoável de eventos, ainda assim acaba limitando-se em não contemplar alguns EVE que possam ter relevância.

Por se tratar de entrevista acompanhada de uma escala de eventos, é importante um rapport apropriado e que propicie ao respondente um ambiente empático e de acolhimento para que assim a obtenção dos dados seja mais enriquecedora. Recomenda-se, por fim, que a utilização deste instrumento em ambiente de pesquisa seja acompanhada de um índice de concordância entre aplicadores, uma vez que o instrumento depende da variabilidade individual do entrevistador.

\section{Referências}

Angst, J., Gamma, A., Rössler, W., Ajdacic, V. \& Klein, D. (2011). Childhood adversity and chronicity of mood disorders. European Archives of Psychiatry and Clinical Neurosciences, 261(1), 21-7.

Arseneault, L., Cannon, M., Fisher, H. L., Polanczyk, G., Moffitt, T. E. \& Caspi, A. (2011). Childhood trauma and children's emerging psychotic symptoms: a genetically sensitive longitudinal cohort study. Am. J. Psychiatry. 168(1), 65-72. doi: 10.1176/appi.ajp.2010.10040567.

Banez, G. A. \& Compas, B. E. (1990). Children's and parents' daily stressful events and psychological symptoms. Journal of Abnormal Child Psychology, 18(6), 591-605.

Bendall, S., Alvarez-Jimenez, M., Hulbert, C. A., McGorry, P. D., Jackson, H. J. (2012). Childhood trauma increases the risk of post-traumatic stress disorder in response to first-episode psychosis. Aust. N. Z. J. Psychiatry, 46(1), 35-9. doi: 10.1177/0004867411430877.

Brown, D. W., Anda, R. F., Tiemeier, H., Felitti, V. J., Edwards, V.J., Croft, J.B. \& Giles, W.H. (2009). Adverse childhood experiences and the risk of premature mortality. American Journal of Preventive Medicine, 37(5), 389-96.

Brown, G. W., Sklair, F., Harris, T. O. \& Birley, J. L. T. (1973). Life-events and psychiatric disorders: Some methodological issues. Psychological Medicine, 3(1), 74-87.

Carrion, V. G., Weems, C. F. \& Reiss, A. L. (2007). Stress predicts brain changes in children: a pilot longitudinal study on youth stress, posttraumatic stress disorder, and the hippocampus. Pediatrics, 119(3), 509-16.

Carter, J.S., Garber, J., Ciesla, J.A. \& Cole, D. A. (2006). Modeling relations between hassles and internalizing and externalizing symptoms in 
adolescents: a four-year prospective study. J. Abnorm Psychol, 115(3), 428-42.

Coddington, R. D. (1972a). The signifance of life events as etiologic factors in the diseases of children. I. A survey of professional workers. Journal of Psychosomatic Research, 16(1), 7-18.

Coddington, R. D. (1972b). The significance of life events as etiologic factors in the diseases of children. II. A study of a nomal population. Journal of Psychosomatic Research, 16(3), 205-13.

Copeland, W. E., Keeler, G., Angold A. \& Costello E. (2007). Traumatic events and posttraumatic stress in childhood. Archives of General Psychiatry, 64(5), 577-84.

Costello, E. J., Edelbrock, C. S. \& Costello, A. J. (1985). Validity of the NIMH Diagnostic Interview Schedule for Children: a comparison between psychiatric and pediatric referrals. Journal of Abnormal Child Psychology, 13(4), 579-95.

Crowe, S. L. \& Blair, R. J. (2008). The development of antisocial behavior: what can we learn from functional neuroimaging studies? Development Psychopathology, 20(4), 1145-59.

Danese, A., Moffitt, T.E., Harrington, H., Milne, B.J., Polanczyk, G., Pariante, C.M., Poulton, R., Caspi, A. (2009) Adverse childhood experiences and adult risk factors for age-related disease: depression, inflammation, and clustering of metabolic risk markers. Arch Pediatr Adolesc Med., 163(12), 1135-43. doi: 10.1001/archpediatrics.2009.214.

De Bellis, M. D. \& Van Dillen, T. (2005). Childhood post-traumatic stress disorder: an overview. Child and Adolescent Psychiatry Clinical North American 14(4), 745-72, ix.

De Bellis, M. D., Hooper, S. R., Woolley, D. P., \& Shenk, C. E. (2010). Demographic, maltreatment, and neurobiological correlates of PTSD symptoms in children and adolescents. Journal of Pediatrics Psychology, 35(5), 570-7.

Dohrenwend, B. P. (2006). Inventorying stressful life evebts as risk factors for psychopatology: toward resolution of the problem of intracategory variability. Psychological Bulletin, 132(3), 477-495.

Flouri, E. \& Panourgia, C. (2011). Adverse life events and emotional and behavioral problems in adolescence: the role of non-verbal cognitive ability and negative cognitive errors. Journal of Abnormal Child Psychology, 39(5), 695-709.

Horesh, N. \& Iancu, I. (2010). A comparison of life events in patients with unipolar disorder or bipolar disorder and controls. Compreensive Psychiatry 51(2), 157-64.
Hulley, S.B., Cummings, S. R., Browner, W. S., Grady, D., Hearst, N., Newman, T. B. (2001). Designing clinical research: an epidemiologic approach. $2^{2}$ ed. Baltimore: Williams \& Wilkins.

Johnson, J. H., \& McCutcheon, S. M, (1980). Assessing life stress in older children and adolescents: preliminary findings with Life Events Checklist. Em: Sarasin, I. G. \&Spielberger, C. D. (Eds.). Stress and Anxiety. Hemisphere, Washington,DC.

Jorge, M. R. (2000). Adaptação transcultural de instrumentos de pesquisa em saúde mental. Em: C. Gorenstein, L. H. S. G. Andrade, A.W. Zuardi, (Eds.). Escalas de avaliação clínica em psiquiatria $e$ psicofarmacologia. (pp. 53-8) São Paulo: Lemos;.

Kashani, J. H., Vaidya, A. F., Soltys, S. M., Dandoy, A. C. \& Reid, J. C. (1990). Life events and major depression in a sample of inpatient children. Compreensive Psychiatry, 31(3), 266-74.

Kaufman, J., Birmaher, B., Brent D, Rao U, Flynn C, Moreci P, Ryan N. (1997). Schedule for Affective Disorders and Schizophrenia for SchoolAge Children-Present and Lifetime Version (KSADS-PL), initial reliability and validity data. Journal of American Academy of Child and Adolescent Psychiatry, 36(7), 980-8.

Kieling, C., Baker-Henningham, H., Belfer, M., Conti, G., Ertem, I., Omigdobun, Rahman, A. (2011). Child and adolescent mental health worldwide: evidence for action. Lancet, 378(9801), 1515-25.

Mayer, L., Lopez-Duran, N., Kovacs M, George C., Baji I, Kapornai K, Vetró A. (2009). Stressful life events in a clinical sample of depressed children in Hungary. Journal of Affective Disorders, 115(1-2), $207-$ 14.

Pasquali, L. (2000). Princípios de elaboração de escalas psicológicas. Em: C. Gorenstein, L. H. S. G. Andrade, A. W. Zuardi, (Eds.). Escalas de avaliação clínica em psiquiatria e psicofarmacologia. (pp. 15-21) São Paulo: Lemos.

Pechtel, P. \& Pizzagalli, D. A. (2011). Effects of early life stress on cognitive and affective function: an integrated review of human literature. Psychopharmacology (Berl), 214(1), 55-70.

Pine, D. S. (2007). Research review: a neuroscience framework for pediatric anxiety disorders. Journal of Child Psychology Psychiatry, 48(7), 631-48.

Pine, D. S., Cohen, P., Johnson, J. G \& Brook, J. S. (2002). Adolescent life events as predictors of adult depression. Journal of Affective Disorders, 68(1), 49-57.

Polanczyk, G., Caspi, A., Williams, B., Price, T.S., Danese, A., Sugden, K., Uher, R., Poulton, R., Moffitt, T. E. (2009). Protective effect of CRHR1 gene variants on the development of adult 
depression following childhood maltreatment: replication and extension. Arch. Gen. Psychiatry, 66(9) 978-85.

doi: 10.1001/archgenpsychiatry.2009.114.

Romero, S., Birmaher, B., Axelson, D. A., Iosif, A. M., Williamson, D. E., Gill, M. K., Keller, M. (2009). Negative life events in children and adolescents with bipolar disorder. Journal of Clinical Psychiatry, 70(10), 1452-60.

Salum, G. A., Polanczyk, G. V., Miguel, E. C., Rohde, L. A. (2010). Effects of childhood development on late-life mental disorders. Curr Opin Psychiatry, 23(6), 498-503. doi: 10.1097/YCO.0b013e32833ead33.

Sar, V., Taycan, O., Bolat, N., Ozmen, M., Duran, A., Oztürk, E. \& Ertem-Vehid, H. (2010). Childhood trauma and dissociation in schizophrenia. Psychopathology, 43(1), 33-40.

Shonkoff, J. P., Boyce, W. T \& McEwen, B. (2009). Neuroscience, molecular biology, and the childhood roots of health disparities: building a new framework for health promotion and disease prevention. JAMA, 301(21), 2252-9.

Williamson, D. E., Birmaher, B., Anderson B. P., AlShabbout M. \& Ryan N. D. (1995). Stressful life events in depressed adolescents: the role of dependent events during the depressive episode. Journal of American Academy of Child and Adolescent Psychiatry, 34(5), 591-8.

Williamson, D. E., Birmaher, B., Dahl, R, E. \& Ryan N. D. (2005). Stressful life events in anxious and depressed children. Journal of Child and Adolescent Psychopharmacology, 15(4), 571-80.

Williamson, D. E., Birmaher, B., Frank E., Anderson B. P., Matty M. K. \& Kupfer D. J. (1998). Nature of life events and difficulties in depressed adolescents. Journal of American Academy of Child and Adolescent Psychiatry, 37(10), 1049-57.

Williamson, D. E., Birmaher, B., Ryan N. D., Shiffrin T. P., Lusky J. A., Protopapa J., Brent D. A. (2003). The stressful life events schedule for children and adolescents: development and validation. Psychiatry Research, 119(3), 225-41.

Recebido em 09/10/2012

Reformulado em 06/02/2013

Aprovado em 26/03/2013 
Nota dos autores:

Apoio Institucional - CNPq e FIPE/ HCPA

Sobre os autores:

Cristian Patrick Zeni é bolsista de Pós-Doutorado Júnior do CNPq, psiquiatra da Infância e Adolescência, mestre e doutor em Psiquiatria pela Universidade Federal do Rio Grande do Sul (UFRGS). É coordenador de pesquisas do Programa para Crianças e Adolescentes com Transtorno Bipolar do Hospital de Clínicas de Porto Alegre.

Roberta Paula Schell Coelho é bolsista de Doutorado pela CAPES do Grupo Neurociência Cognitiva do Desenvolvimento do Programa de Pós-Graduação em Psicologia da Pontifícia Universidade Católica do Rio Grande do Sul (PUCRS), psicóloga e mestre em Psicologia pela PUCRS.

Aline Aparecida Martins Ferreira é psicóloga formada pela PUCRS e auxiliar de pesquisa no Grupo Neurociência Cognitiva do Desenvolvimento do Programa de Pós-Graduação em Psicologia da PUCRS.

Priscila de Oliveira Machado é psicóloga formada pela PUCRS e auxiliar de pesquisa no Grupo Neurociência Cognitiva do Desenvolvimento do Programa de Pós-Graduação em Psicologia da PUCRS.

Silzá Tramontina é psiquiatra da Infância e Adolescência, mestrado em Medicina: Ciências Médicas pela UFRGS, e doutora em Psiquiatria pela UFRGS, e coordenadora do Programa de Crianças e Adolescentes Bipolares (ProCAB). Médica contratada do Hospital de Clínicas de Porto Alegre.

Rodrigo Grassi-Oliveira é bolsista Produtividade $2 \mathrm{CNPq}$, professor do Programa de Pós-Graduação em Psicologia da PUCRS e coordenador do Grupo Neurociência Cognitiva do Desenvolvimento. É psiquiatra com Mestrado e Doutorado em Psicologia pela PUCRS e pós-doutorado em Psicobiologia e Epigenética no Queensland Brain Institute (QBI) da The University of Queensland.

Contato com os autores:

Pontifícia Universidade Católica do Rio Grande do Sul Av. Ipiranga, 6.681 - Partenon - CEP 90619-900 - Porto Alegre-RS, Brasil E-mail: rodrigo.grassi@pucrs.br 